\title{
E-MAIL COUNSELING FOR THE ULTRA-ORTHODOX COMMUNITY
}

\author{
Osnat Rubin \\ Counseling and Human Development, University of Haifa (Israel)
}

\begin{abstract}
This study examines a unique project of its kind among the ultra-Orthodox community, in which ultra-Orthodox M.A. students consult via e-mail to members of the community. The procedure is anonymous: the client's personal email details are stored in the system, students are given the content of the request without the identifying information; and they write a reply, which is transmitted through the system to the client, within a week at the most. Counseling model includes one answer via e-mail, with no further interaction.

The advantages of using e-mail as a medium for counseling include: therapeutic aspects of writing, the ability to maintain anonymity on the part of the client, asynchronous that allows the consultant to plan, design, correct, re-examine and save the text, as well as the independence of time and place for consultation process.

Psychotherapy or mental health care in the ultra-Orthodox sector involves shame, reluctance and resistance. An ultra-Orthodox applying psychological help within this enclave community, risks a negative label, both regarding himself and his family. Thus, the anonymity that characterizes the online counseling may be a solution and may have an additional value as first-aid for those who avoid turning to face-to-face help.

The present study analyzed a total of 60 cases of e-consultation within this project. Five stages were identified in the process, as well as unique aspects of e-counseling in the ultra-Orthodox sector. These findings will be discussed in light of counseling literature and methods of discourse analysis.
\end{abstract}

Keywords: E-counseling, ultra-orthodox, credibility, faith.

\section{References}

Rubin, O. (2017). "Good advice": e-mail consultation to the ultra-Orthodox sector. Efshar: Social Work Journal, 28, 39-42. [Hebrew]. 